\title{
Matter-Enhanced Neutrino Oscillations in the Quasi-Adiabatic Limit
}

\author{
A. B. Balantekin ${ }^{1,2,3 *}$, J. F. Beacom ${ }^{1 \dagger} \ddagger$ and J. M. Fetter ${ }^{1 \S}$ \\ ${ }^{1}$ Department of Physics, University of Wisconsin \\ Madison, Wisconsin 53706 USA \\ ${ }^{2}$ Institute for Nuclear Theory, University of Washington, Box 351550 \\ Seattle, WA 98195-1550 USA \\ ${ }^{3}$ Department of Astronomy, University of Washington, Box 351580 \\ Seattle, WA 98195-1580 USA
}

(August 29, 2018)

\begin{abstract}
We introduce simple analytic expressions for the neutrino survival probability in media valid in the quasi-adiabatic limit. These expressions provide a quick but accurate alternative to numerical solution of the neutrino propagation equations for the Mikheyev-Smirnov-Wolfenstein effect. They can also be used to extract information about the density scale height from the neutrino data. As an example, we present calculations for solar neutrinos.
\end{abstract}

14.60.Pq, 96.60.Jw, 26.65.+t

*Electronic address: baha@nucth.physics.wisc.edu

$\dagger$ Electronic address: beacom@citnp.caltech.edu

†Present address: Physics 161-33, Caltech, Pasadena, CA 91125 USA

$\S$ Electronic address: fetter@nucth.physics.wisc.edu 
Neutrino flavor conversion via the Mikheyev-Smirnov-Wolfenstein (MSW) mechanism [1] has been studied in various astrophysical environments, most extensively in the sun [2.3] and supernovæ [i] . It is straightforward to solve the appropriate equations of motion analytically in some special cases [5], and numerically for an arbitrary density profile. However, as one is treating a quantum-mixing problem over considerable distances, calculations which search a broad range of parameter space may quickly become more tedious than this fundamentally simple level-crossing problem should warrant. Furthermore, analytic approximations are usually sufficient to highlight the salient physics.

A convenient starting point for most approximations is the formula [2] for the averaged survival probability of a given neutrino flavor:

$$
P_{\nu}(E)=\frac{1}{2}\left[1+\left(1-2 P_{h o p}\right) \cos 2 \theta_{v} \cos 2 \theta_{i}\right] .
$$

In this equation the vacuum- and matter-mixing angles are introduced in the usual way, twoflavor mixing is assumed, and $P_{h o p}$ denotes the probability of hopping from one adiabatic eigenstate to the other. One typically averages over the initial matter angle $\cos 2 \theta_{i}$ over the region of neutrino production. An excellent approximation for the hopping probability is the quasi-adiabatic expression

$$
\begin{aligned}
P_{\text {hop }} & =\exp (-\pi \Omega), \\
\Omega & =\frac{i}{\pi} \frac{\delta m^{2}}{2 E} \int_{r_{0}}^{r_{0}^{*}} d r\left[\zeta^{2}(r)-2 \zeta(r) \cos 2 \theta_{v}+1\right]^{1 / 2},
\end{aligned}
$$

where $r_{0}^{*}$ and $r_{0}$ are the turning points (zeros) of the integrand. In this expression we have defined

$$
\zeta(r)=\frac{2 \sqrt{2} G_{F} N_{e}(r)}{\delta m^{2} / E},
$$

where $N_{e}$ is the number density of electrons in the medium. By analytic continuation, this complex integral is primarily sensitive to densities near the resonance point. $P_{h o p}$ varies slightly over the production region, since neutrinos produced at different places see slightly different variations of density along their paths. When the full three-dimensional geometry of the production region is taken into account, this is a small but nonzero effect. For purposes of demonstration, however, we will neglect the variation of $P_{h o p}$ with production point.

Eq. (目) for $P_{\text {hop }}$ is valid for an arbitrary density profile, and for a large range of mixing parameters. In Ref. [6], a uniform semiclassical solution of the MSW equations was introduced, and solved numerically. In Ref. [0], a complete analytic solution for the oscillation probability (including interference terms) was given, and the expression for $P_{h o p}$ above derived as a consequence. Using a different technique, the form of $P_{h o p}$ above was found for the exponential density in Ref. [8], and for the general case in Ref. [9]. It is an excellent approximation from the adiabatic regime up to the extreme non-adiabatic limit [7]. In particular, it has a larger range of validity than the linear Landau-Zener result, which can be recovered as a special case of Eq. (2).

For the small-angle solution to the solar neutrino anomaly, the propagation is nonadiabatic in the energy range of interest. $P_{h o p}$ is appreciable (but not maximal) and is thus 
well-approximated by Eq. 2. For this solution, $\cos 2 \theta_{i}=-1$ to a very good approximation, so we have a complete analytic solution for $P_{\nu}(E)$. The resonance condition

$$
\frac{\delta m^{2}}{E} \cos 2 \theta_{v}=2 \sqrt{2} G_{F} N_{e}\left(r_{\text {res }}\right)
$$

relates the density probed by the resonance to a given neutrino energy. In Figure 1(a), we show the density profile from the 1995 standard solar model (SSM) of Bahcall and Pinsonneault [10]. For the small angle solution, the resonance for a $5 \mathrm{MeV}$ neutrino occurs at about $0.35 R_{\odot}$; for a $15 \mathrm{MeV}$ neutrino it occurs at at about $0.45 R_{\odot}$. In that region, the solar density is approximately exponential. This form of the density motivates an expansion of the electron number density scale height, $r_{s}$, in powers of density:

$$
-r_{s} \equiv \frac{N_{e}(r)}{N_{e}^{\prime}(r)}=\sum_{n} b_{n} N_{e}^{n}
$$

where prime denotes derivative with respect to $r$. In this expression a minus sign is introduced because we assumed that density profile decreases as $r$ increases. For an exponential density profile only the $n=0$ term is present. The $n \neq 0$ terms represent deviations from the exponential profile. The density scale height is more sensitive to the fine structure of the medium than the density profile itself. Even for a density profile which appears nearly exponential, the scale height may vary significantly from a constant value. To illustrate this we plot in Figure 1(b) the density scale height for the standard solar model. In principle, for most monotonic density profiles the scale height can be fitted using an expansion as given in Eq. (5). One should emphasize that, for an arbitrary density profile, the expansion in Eq. (5) is not a perturbative expansion. That is, one should not expect the coefficients $b_{n}$ to necessarily decrease as $n$ increases. Fits truncated in a given order could result in coefficients which are rather different from coefficients obtained with a different order, much like fitting a function with different order splines.

Eq. (5) can easily be integrated to yield

$$
r\left(N_{e}\right)=r_{b}+b_{0} \log N_{e}+\sum_{n \neq 0} \frac{b_{n}}{n} N_{e}^{n},
$$

where $r_{b}$ is an integration constant, i.e., a set of $b_{n}$ 's specifies $N_{e}(r)$ up to a radial shift. Hence if only one of the $b_{n}$ 's in this expansion were non-zero, it would lead to $N_{e}(r) \sim r^{1 / n}$ for $n>0$, and $N_{e}(r) \sim(1 / r)^{1 /|n|}$ for $n<0$. In principle, such $n<0$ terms could appear, but they seem to be more singular than necessary for modeling the solar density, hence in the rest of this paper we take $n \geq 0$. In practice one would fit a given density profile using a finite number of terms, as illustrated in Figure 2. The fit is only to be made over the range of densities probed via Eq. 4 and the given range of energy. In this figure we show the density scale height of the standard solar model in the region probed by the Sudbury Neutrino Observatory (SNO) [11] and SuperKamiokande (SK) [12 detectors. We then fit it

\footnotetext{
${ }^{1}$ But they may be necessary in other cases, e.g., for the density profile in a core-collapse supernova, where this method is equally applicable.
} 
by truncating the expansion in Eq. (5) at the $n=0,1$, and 2 term, respectively. Just a few terms of the series approximate the model's density profile quite well.

Inserting the expansion of Eq. (5) into Eq. (2), and using an integral representation of the Legendre functions [13], one obtains

$$
\begin{aligned}
\Omega & =-\frac{\delta m^{2}}{2 E}\left\{b_{0}\left(1-\cos 2 \theta_{v}\right)\right. \\
& \left.+\sum_{n=1}^{\infty}\left(\frac{\delta m^{2}}{2 \sqrt{2} G_{F} E}\right)^{n} \frac{b_{n}}{2 n+1}\left[P_{n-1}\left(\cos 2 \theta_{v}\right)-P_{n+1}\left(\cos 2 \theta_{v}\right)\right]\right\},
\end{aligned}
$$

where $P_{n}$ is the Legendre polynomial of order $\mathrm{n}$. The first term in Eq. (17) represents the contribution of the exponential density profile alone (as was implicit in the treatment in Ref. [14]). Eq. (7) is remarkable in that it directly connects an expansion of the logarithm of the hopping probability in powers of $1 / E$ to an expansion of the density scale height. That is, after a slight amount of computation, it provides a direct connection between $N_{e}(r)$ and $P_{\nu}\left(E_{\nu}\right)$. Consequently these equations provide a quick and accurate alternative to numerical integration of the MSW equation for any density profile, for a wide range of mixing parameters. To illustrate the utility of Eq. (7) in Figure 3, we compare the electron neutrino survival probability in the Sun obtained using this equation with the exact numerical calculation. To generate the approximate survival probabilities in this figure, we used the fit coefficients from Figure 2 and computed $\cos 2 \theta_{i}$ numerically. Eq. (7) is an excellent approximation; the relative error in $P_{\nu}$ is well under $1 \%$ for the order 2 fit, across the full range of neutrino energy probed by SNO and SK. We fit the $r_{s}$ profile only for densities where neutrinos in our energy range undergo resonance.

The SNO and SK detectors are sensitive to the spectrum distortion due to the MSW effect. In Figure 4, we calculate the spectrum distortion for the small angle MSW solution at SNO, using the method of Ref. 15] and neglecting backgrounds. For the calculation of the neutrino-deuterium charged-current cross-sections we used the code of Bahcall and Lisi [16]. This encoded differential charged-current cross-section is based on the Ellis-Bahcall effective range calculation [17] with slight improvements. One observes that even the first term in Eq. (7) - that is, the pure exponential - is sufficient to describe five years of data collection, and to show the distortion from the no-oscillation spectrum. The success of using a single term depends, however, on the use of the correct scale height in the range of resonance densities probed by MSW. This scale height may be quite different from the scale height that fits the entire sun with an exponential $N_{e}(r)$. For our mixing parameters, the fit to the full sun would give a significantly different result, as noted below.

The MSW effect is an energy- and density-dependent effect. With the analytic forms given above, this is explicit for an arbitrary density profile. From the measured energy dependence of neutrino data, one can use these expressions to invert for the scale height as a function of density and hence density as a function of radius (up to a radial shift). Data from SNO, in principle, would be particularly suitable for such an analysis for two reasons. First, the electron spectra is the charged-current is sharply peaked at the neutrino energy. Second, it may be possible to use the neutral-current measurement to determine total ${ }^{8} \mathrm{~B}$ solar neutrino flux. The range of densities probed depends on the range of energies probed, via Eq. 4. As noted above, an energy range of $E=5-15 \mathrm{MeV}$ probes a radial range of approximately $0.35 R_{\odot}-0.45 R_{\odot}$ if the small-angle solution to the solar neutrino anomaly 
is assumed. These resonance positions are far outside the region where the high-energy ${ }^{8} \mathrm{~B}$ neutrinos are produced (peak production is at approximately $0.05 R_{\odot}$ ). To a large extent, the finite size of the source can be ignored, and only the energy distribution of these neutrinos is needed. This is not exact, as neutrinos created in a finite spherical source region and traveling to the earth will see slightly different variations of density along their paths, and will hence have slightly different hopping probabilities. The primary systematic limitations of this inversion are then the accuracy of the analytic form for $P_{h o p}$ and the treatment of a finite source region.

However, the strongest limitation to making such an inversion is the statistical error. Thus probing the solar interior via the MSW effect will require a sensitivity that goes beyond that of current solar neutrino detectors, as we discuss below. In Figure 5, we show what SNO may accomplish with regard to inversion in five years of running time. To obtain the confidence regions in this figure, we predicted the electron energy spectrum from chargedcurrent deuteron breakup at SNO after 5 years of counting. We found the electron neutrino survival probabilities by full-fledged numerical integration of the MSW equation through the standard solar model of Bahcall and Pinsonneault. Again, we used the method of Ref. [15, which takes account of SNO's finite electron energy resolution. We then randomly generated 2000 electron energy spectra, Poisson-distributed about the theoretical expectation. We fit each of the 2000 statistical instances with Eq. (7), and used that the fit coefficients to produce 2000 measured scale height profiles. Then the CL\% confidence region in the figure encloses the measured profiles for the CL\% of sample runs whose spectra, before fitting, have the smallest $\chi^{2}$ with respect to the original spectrum.

Figure 5 a shows the results of this procedure when we truncated the series in Eq. (7) after $n=0$, that is, we treated the electron density profile as a pure exponential. In Figure 5b we took account of first-order deviations from exponential, truncating the series after $n=1$. In both figures, the solid line shows the scale height as a function of density in the SSM, while the long-dashed line in the center shows the result of fitting the original spectrum. Where the solid line goes outside the confidence regions in Figure 5a, it simply means that we are not very sensitive to the scale height at those densities. Figure $5 b$ clearly shows the region of sensitivity; we can determine the scale height with some accuracy around $2 \times 10^{24} \mathrm{~cm}^{-3}$, but not elsewhere. This is where neutrinos of energy near $10 \mathrm{MeV}$ undergo resonance, and those neutrinos contribute the most to the observed electron spectrum.

Quite different scale-height profiles can give rise to rather similar survival probabilities. This makes inversion a particularly challenging problem as the size of the confidence regions in the figure illustrates. Note, however, that we can easily rule out the value of scale height which fits the entire sun as an exponential, $6.6 \times 10^{9} \mathrm{~cm}$.

In conclusion, we have presented simple analytic expressions for the neutrino survival probability in media. These expressions where the energy dependence of the hopping probability is related to the density-scale height in the medium are valid in the quasi-adiabatic limit. To demonstrate the technique for the solar neutrinos, we neglected effects arising from the three-dimensional neutrino source geometry. However, one may easily incorporate them by fitting the density profile separately for each ray from the neutrino production region to the earth. Since ordinarily one must numerically integrate the MSW equations for each ray, this technique still vastly speed up the computation of $P_{\nu}$. As long as the density profile is monotonic in the resonance region and is not subject to fluctuations such as those described 
in Ref. [18], Eq. (7) provides a quick and accurate alternative to numerical solution of the neutrino propagation equations for the Mikheyev-Smirnov-Wolfenstein effect. Its validity is not necessarily restricted to the Sun; they can also be used to calculate the MSW effect for type II supernovæ and the day-night effect in Earth. Used in reverse, these approximations in principle allow one to invert neutrino data for the density profile. However, the statistics of the current and near-term neutrino detectors are not adequate to do this in a precise way.

\section{ACKNOWLEDGMENTS}

We thank members of the University of Washington SNO group for useful discussions and J.N. Bahcall and E. Lisi for permission to use their computer code for the calculation of the neutrino-deuterium cross sections. This work was supported in part by the U.S. National Science Foundation Grant No. PHY-9605140 at the University of Wisconsin, and in part by the University of Wisconsin Research Committee with funds granted by the Wisconsin Alumni Research Foundation. We thank the Department of Energy's Institute for Nuclear Theory and Department of Astronomy at the University of Washington for their hospitality and Department of Energy for partial support during the completion of this work. J.F.B. thanks Caltech for support as a Sherman Fairchild Postdoctoral Scholar in Physics during the final stages of this work. 


\section{REFERENCES}

[1] S.P. Mikheyev and A. Yu. Smirnov, Sov. J. Nucl. Phys. 42 (1985) 913; Sov. Phys. JETP 64 (1986) 4; L. Wolfenstein, Phys. Rev. D 17 (1978) 2369; Phys. Rev. D 20 (1979) 2634.

[2] H. Bethe, Phys. Rev. Lett. 56 (1986) 1305; W.C. Haxton, Phys. Rev. Lett. 57 (1986) 1271; S.J. Parke, Phys. Rev. Lett. 57 (1986) 1275.

[3] S.P. Rosen and J.M. Gelb, Phys. Rev. D 34 (1986) 969; V. Barger, R.J.N. Phillips, and K. Whisnant, Phys. Rev. D 34 (1986) 980; W.C. Haxton, Phys. Rev. D 35 (1987) 2352; J.N. Bahcall and H.A. Bethe, Phys. Rev. Lett. 65 (1990) 2233.

[4] G.M. Fuller, R.W. Mayle, B.S. Mayer, and J.R. Wilson, Astrophys. J. 389 (1992) 517; S.E. Woosley and R. Hoffman, ibid. 395 (1992) 202; S.E. Woosley, G.J. Mathews, J.R. Wilson, R.D. Hoffman, and B.S. Meyer, ibid. 433 (1994) 229; G.M. Fuller, Phys. Rep. 227 (1993) 149; Y.-Z. Qian, G.M. Fuller, G.J. Matthews, R.W. Mayle, J.R. Wilson, and S.E. Woosley, Phys. Rev. Lett. 71 (1993) 1965; F.N. Loreti, Y.-Z. Qian, G.M. Fuller, and A.B. Balantekin, Phys. Rev. D 52 (1995) 6664.

[5] A.B. Balantekin, hep-ph/9712304.

[6] A.B. Balantekin, S.H. Fricke, and P.J. Hatchell, Phys. Rev. D 38 (1988) 935.

[7] A.B. Balantekin and J.F. Beacom, Phys. Rev. D 54 (1996) 6323.

[8] P. Pizzochero, Phys. Rev. D 36 (1987) 2293.

[9] T. Kuo and J. Pantaleone, Phys. Rev. D 39 (1989) 1930.

[10] J.N. Bahcall and M.H. Pinsonneault, Rev. Mod. Phys. 67 (1995) 781.

[11] SNO Collaboration, G. Aardsma et al., Phys. Lett. B 194 (1987) 321.

[12] Y.Totsuka, to be published at the Proceedings of the Lepton Photon Symposium $199 \%$.

[13] E.T. Whittaker and G.N. Watson, A Course of Modern Analysis (Cambridge, New York, 1973), p. 315.

[14] W. Kwong and S.P. Rosen, Phys. Rev. D 51 (1995) 6159.

[15] J. N. Bahcall, P. I. Krastev, and E. Lisi, Phys. Rev. C 55 (1997) 494.

[16] J.N. Bahcall and E. Lisi, Phys. Rev. D 54 (1996) 5417. See also http://www.sns.ias.edu/ jnb/sNdata/deuteriumcross.html.

[17] S.D. Ellis and J.N. Bahcall, Nucl. Phys. A 114 (1968) 636.

[18] F.N. Loreti and A.B. Balantekin, Phys. Rev. D 50 (1994) 4762; A.B. Balantekin, J. M. Fetter, and F. N. Loreti, Phys. Rev. D 54 (1996) 3941; H. Nunokawa, A. Rossi, V.B. Semikoz, and J.W.F. Valle, Nucl. Phys. B 472 (1996) 495; C.P. Burgess and D. Michaud, Ann. Phys. 256 (1997) 1; E. Torrente-Lujan, hep-ph/9602398. 


\section{Figure Captions}

Figure 1. (a) Density as a function of radius in the 1995 standard solar model of Bahcall and Pinsonneault [10], shown with a solid line. The long-dashed line is the exponential fit over the whole sun. (b) Scale height as a function of radius in the same model, shown with a solid line. The long-dashed line is the exponential fit over the whole sun. The derivatives are obtained by smoothed splines from the tables in Ref. [10.

Figure 2. Scale height as a function of density in the standard solar model, in the region probed by SNO and SK with the mixing parameters $\sin 2 \theta_{v}=0.01$ and $\delta m^{2}=5.0 \times 10^{-6}$ $\mathrm{eV}^{2}$. The solid line is the SSM value. The dashed, dot-dashed, and dotted lines are obtained by using the expansion in Eq. (5) with values of $n$ up to 0, 1, and 2, respectively.

Figure 3. (a) Electron neutrino survival probability in the SSM, as a function of neutrino energy. The neutrino mixing parameters are the same as in Figure 2. The dashed, dotdashed, and dotted lines are obtained from Eq. (7) with values of $n$ up to 0, 1, and 2, respectively, and with the parameters $b_{n}$ determined from the fits in Figure 2. (b) The relative error, (exact - approx) / exact, introduced by using Eq. (7) rather than a full numerical integration.

Figure 4. (a) Spectrum distortion for the small-angle MSW solution at SNO, resulting from the survival probabilities of Figure 3. The solid line is the exact numerical solution. The dashed, dot-dashed, and dotted lines result from values of $n$ up to 0,1 , and 2 , as in Figure 3. The error bars on the exact numerical result correspond to two and five years of data collection. The dot-dot-dot-dashed line is the spectrum without MSW oscillations, normalized to the same total rate as with MSW oscillations. Note that on the scale of this graph the $n=1$ and 2 lines are not distinguishable from the exact answer. (b) The relative error arising from the use of Eq. (7).

Figure 5. (a) A determination of the density scale height in the range of densities probed by MSW after five years of data collection at SNO. The solid line is the scale height as a function of density in the SSM. The long-dashed line in the center of the figure is the scale height which best fits the resulting electron energy spectrum, taking only the $n=0$ term in Eq. (7). The dot-dashed line shows the boundary of the $68 \%$ confidence region for scale height, and the dot-dot-dot-dashed line shows the $99 \%$ confidence region. A dotted line which shows the $90 \%$ region is not visible in the figure; the region's upper limit is the same as the $68 \%$ region, and the lower limit is the same as the $99 \%$ region. (b) As (a), but including the $n=1$ term in Eq. (17). We now see clearly the region of sensitivity in density, which corresponds to the resonance densities for neutrinos that contribute significantly to the observed electron spectrum. 
Figure 1

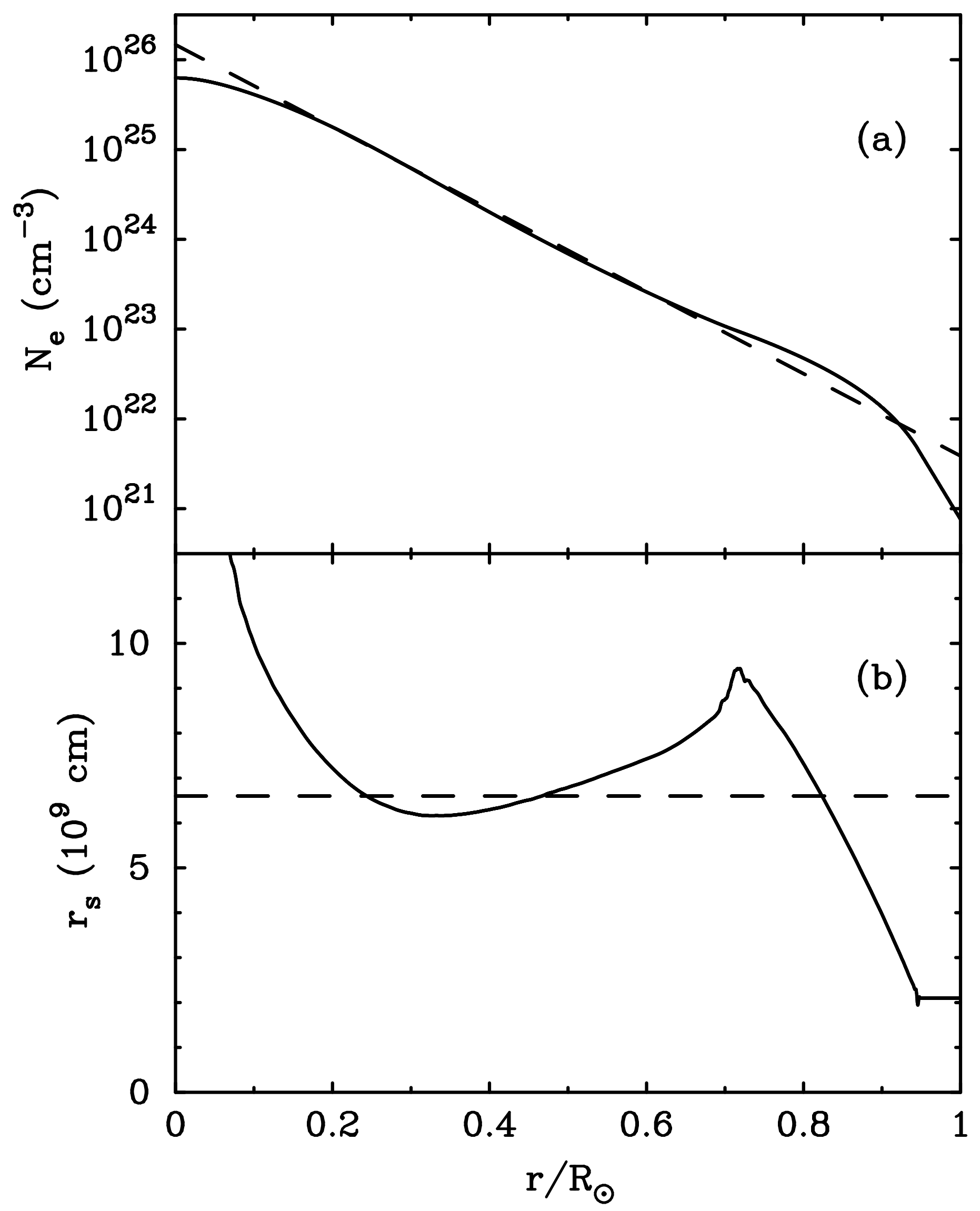




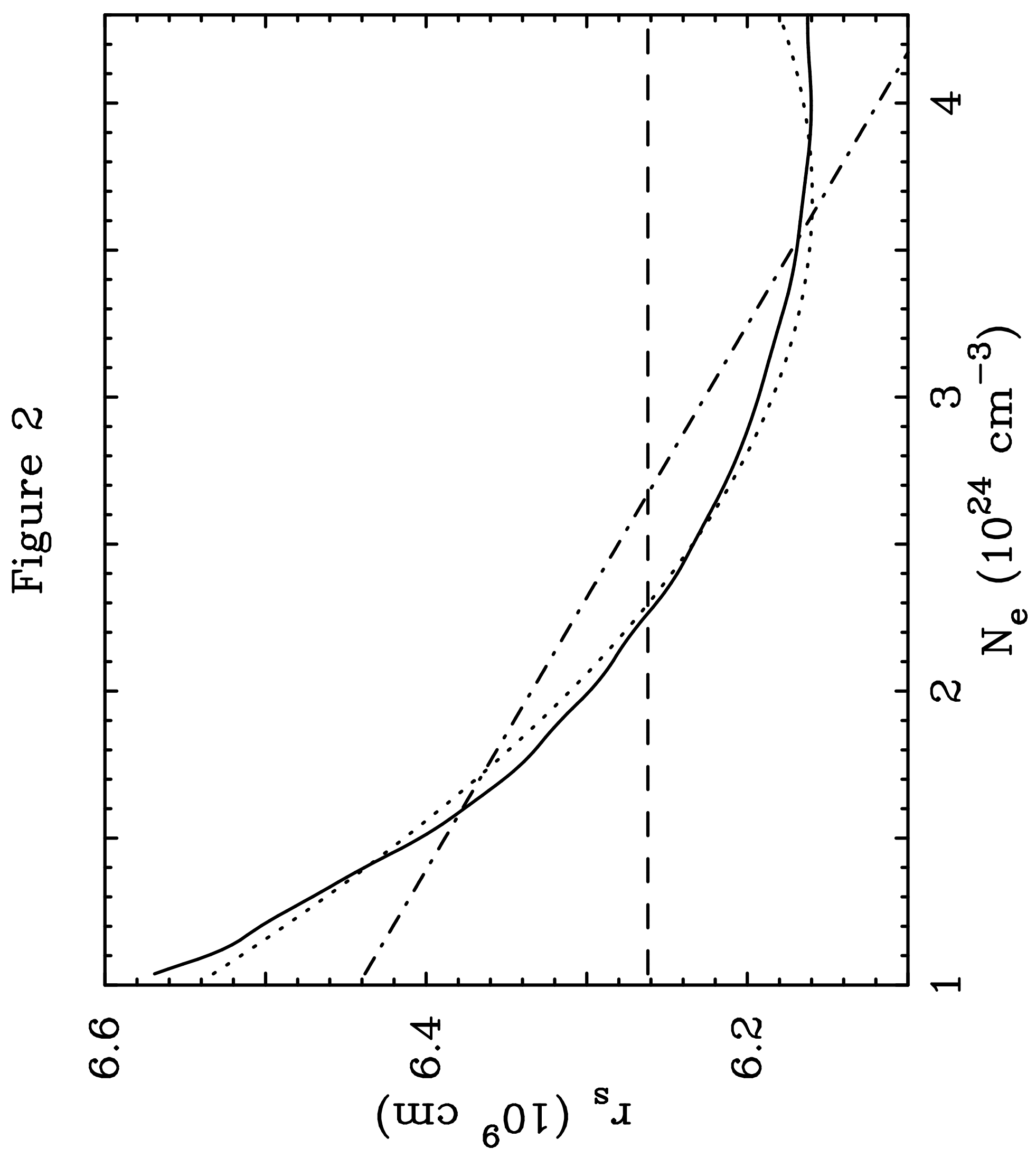


Figure 3

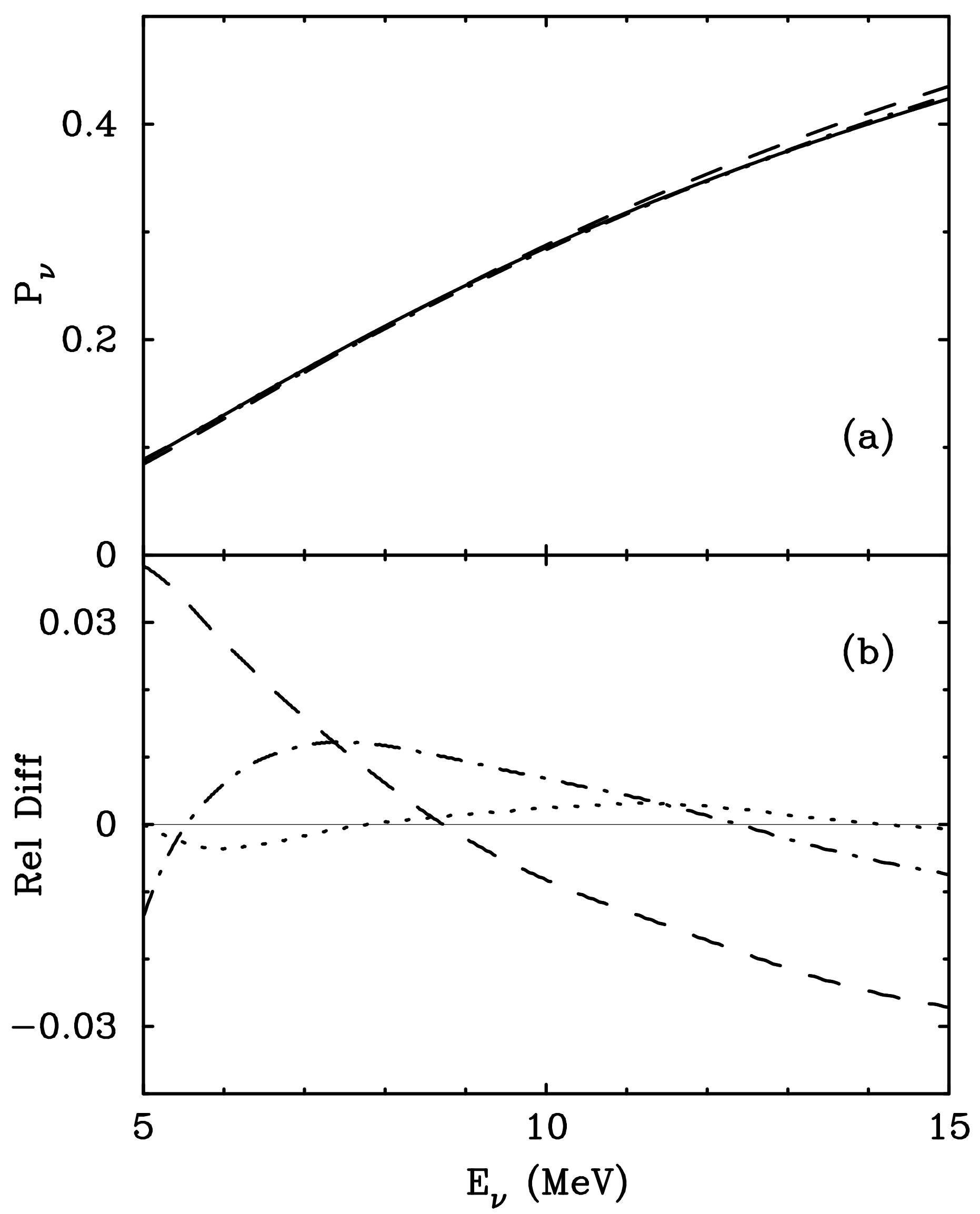


Figure 4

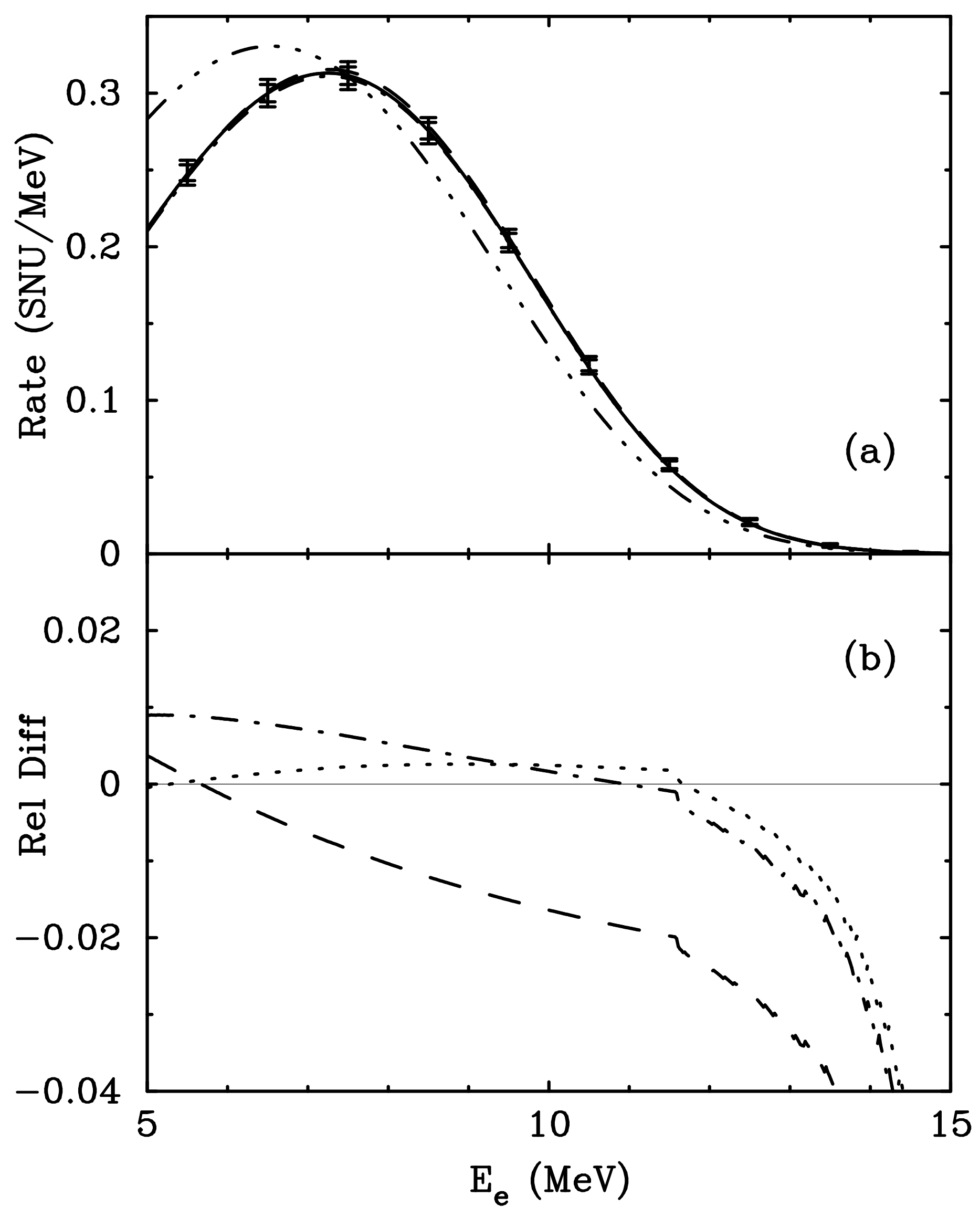


Figure 5

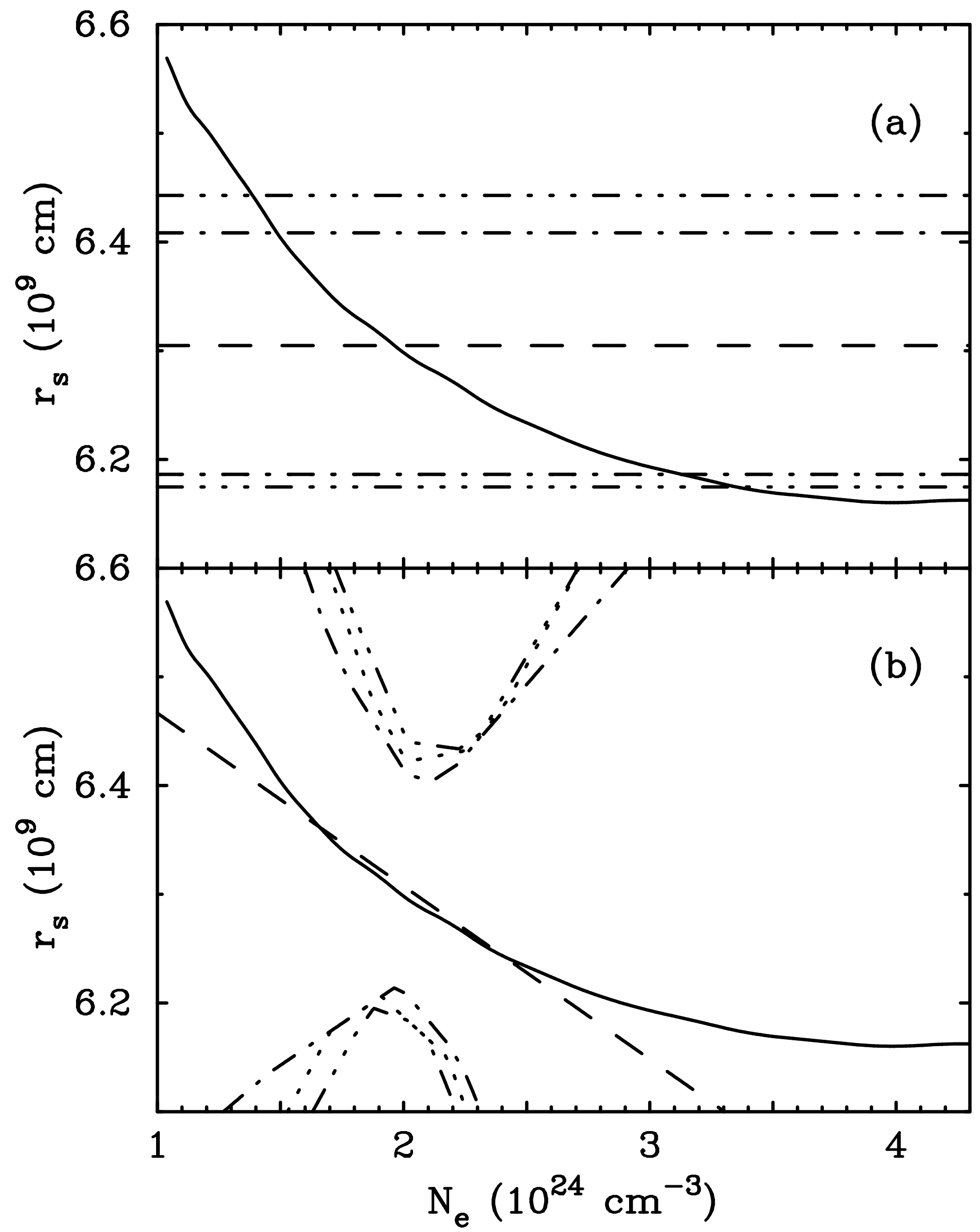

